\title{
Reshaping Hybrid Perovskites Emission with Flexible Polymer Microcavities
}

\author{
Paola Lova, ${ }^{1}$ Paolo Giusto, ${ }^{2}$ Francesco Di Stasio, ${ }^{3}$ Giovanni Manfredi, ${ }^{1,2}$ Giuseppe M. Paternò, ${ }^{2}$ Daniele Cortecchia, ${ }^{2,3}$ \\ Cesare Soci $^{3}$ and Davide Comoretto ${ }^{1 *}$ \\ ${ }^{1}$ Dipartimento di Chimica e Chimica Industriale, Università di Genova, 16146 Genova, Italy. \\ 2 Istituto Italiano di Tecnologia, Center for Nano Science and Technology, 20133 Milano, Italy. \\ 3 Centre for Disruptive Photonic Technologies, TPI, SPMS, Nanyang Technological University, 21 Nanyang Link, Singapore 637371, \\ Singapore d Energy Research Institute@NTU (ERI@N), Nanyang Technological University, 50 Nanyang Drive, Singapore 6375532, \\ Singapore.
}

\begin{abstract}
Thanks to versatile optoelectronic properties solution processable perovskites have attracted increasing interest as active materials in photovoltaic and light emitting devices. However, the deposition of perovskite thin films necessitates wide range solvents that are incompatible with many other solutionprocessable media, including polymers that are usually dissolved by the perovskite solvents. In this work, we demonstrate that hybrid perovskite thin films can be coupled with all polymer planar photonic crystals with different approaches to achieve emission intensity enhancement and reshaping using different approaches. The possibility to control and modify the emission spectrum of a solution processable perovskite via a simple spun-cast polymer structure is indeed of great interest in optoelectronic applications requiring high color purity or emission directionality. Furthermore, thanks to the ease of fabrication and scalability of solution-processed photonic crystals, this approach could enable industrial scale production of low-cost, large area, lightweight and flexible polymer-perovskite lighting devices, which may be tuned without resorting to compositional engineering.
\end{abstract}

\section{Introduction}

Thanks to a high photoluminescence efficiencies, large charge carrier diffusion, and easy solution processing, hybrid organic-inorganic perovskites have been largely investigated for a variety of flexible optoelectronic devices.[1-5] However, their deposition often requires solvents able to dissolve most of commercial polymers, limiting the possibility to couple this active media with flexible polymer devices. For instance, white emitting bidimensional perovskites are very interesting for lightening applications.[6-14] Indeed, coupling this emitters with all polymer structures processed from solution and able of single color emission enhancement or suppression would lead to reduction of fabrication cost and simplification of fabrication processes in lightening devices. On the other hand, the necessity to process the perovskite from broad-spectrum solvents such as dimethyl formamide (DMF) or dimethyl sulfoxide (DMSO), which would dissolve many polymers, makes this perovskite incompatible with polymer processing.

Among photonic structures, solution processed planar systems, also knows ad distributed Bragg Reflectors (DBRs) are interesting thanks to the possibility to fabricate them from solution and the ease of tunability of their optical properties by simple tuning of the processing conditions.[15-17] Beside the low refractive index contrast available for commercial polymers that can be used for the fabrication of these structure, these system have been largely employed for several applications including sensing,[18-21], photon recycling in photovoltaics,[22] and lasing;[23] while fluorescence enhancement and control have been achieved for a variety of polymer,[24] organic,[25] and inorganic[26] media. On the other hand, coupling these polymer structures with solution processable hybrid perovskites is still challenging.

In this work we compare three different approaches that can be used to embed perovskite emitters into planar polymer microcavities and photonic structures. For this purpose, we report on a white emitting perovskite 2,2'(ethylenedioxy)bis(ethylammonium) lead chloride and methylammonium lead iodide perovskite thin film embedded in the photonic structure with different architectures.

\section{Experimental Methods}

2,2'-(ethylenedioxy)bis(ethylammonium) lead chloride ((EDBE) $\mathrm{PbCl}_{4}$ ) and methylammonium lead iodide MA

\footnotetext{
* Corresponding author: davide.comoretto@unige.it
} 
$\mathrm{PbI}_{3}$ hybrid perovskite thin films were synthetized as previously reported.[8,27]

Polymer planar microcavities and DBRs were grown on top of substrates made of fused silica or glass by spincoating of alternated layers of polystyrene (PS, $\mathrm{Mw}=200000$; refractive index, $\mathrm{n}=1.58)$ or $\operatorname{poly}(\mathrm{n}$ vinylcarbazole) ( $\mathrm{PVK} ; \mathrm{Mw}=40000 ; \mathrm{n}=1.68)$ dissolved in toluene and cellulose acetate $(\mathrm{CA}, \mathrm{Mw}=61000, \mathrm{n}=1.46)$. The rotation speed was kept between 4000 and 8000 RPM. Reflectance and photoluminescence spectra were collected as described in previous reports.[8]

\section{Results and Discussion}

The top panels (a, b, and c) of Figure 1 show the schematics of the three architectures used to couple the hybrid perovskite thin films with planar polymer photonic structures. The first one is the simples (Fig. 1a). In this case an (EDBE) $\mathrm{PbCl}_{4}$ film is casted on substrates of fused silica or thick polyethylene terephthalate. A DBR made of PS and CA is successively spun-cast on top of this perovskite film.[8] The reflectance spectrum of the sample surface was collected in 9 spots and is reported in Figure 1a'. Here, we notice that the 9 spectra are superimposable, indicating the homogeneity of the sample. Moreover, all the spectra show a maximum in the reflectance intensity at $510 \mathrm{~nm}$, assiged to the PBG. The spectra are also characterized by an interference pattern which assesses the good optical quality of the sample. For what concern the effect of the photonic structure on the broad perovskite emitter, Figure 1 a" compares the emission spectrum of the bare perovskite thin film (black line) with the one of the photonic structures (red line). The bare (EDBE) $\mathrm{PbCl}_{4}$ emission appears quite broad ranging from $400 \mathrm{~nm}$ to more than $600 \mathrm{~nm}$ and is peaked in the green region of the spectrum. When coupled to the polymer DBR, the emission spectrum is strongly modified. Indeed, the intensity of the emission is enhanced in the entire range due to better light extraction (see also Figure 1 a" '), moreover the emission spectrum result modulated by both the PBG and the interference patter. A Peak of intensity enhancement is detected slightly below $500 \mathrm{~nm}$, Such effect was assigned to a weak coupling regime favoured by the low reflectivity of the substrate.[8]

When the same materials are employed to fabricate a microcavity where the perovskite constitute the defect layer (Figure 1b), the deposition of the perovskite thin film from DMSO solution on the first half microcavity favour the polymer dissolution. Such phenomenon introduces defects, roughness and inhomogeneities within the structure. The reflectance spectra of Figure 1b' show indeed that the sample is not homogeneous, and is characterize by low optical quality, as the interference pattern is barely visible. The PBG, positioned at about $640 \mathrm{~nm}$ is characterized by low reflectance intensity and appears broadened. Moreover, very broad cavity modes are detectable within this signal $(\mathrm{Q} \approx 20)$. The large disorder induced by the deposition of the perovskite layer implies that the photonic structure has only small effects on the perovskite emission. Comparing the emission spectra of Figure 1b", one notice that the emission intensity of the microcavity (red line) results larger with respect to the reference sample (black line) owing again to better light extraction in the microcavity. On the other hand, emission reshaping induced by the photonic structure is barely detectable. Indeed, only a broad relative maximum is visible at about $640 \mathrm{~nm}$ (Compare with Figure 1b"'). Commonly, polymer planar microcavity with structure similar to the one just reported show quality factor up to 250.[28] The low quality of this structure can then be attributed to the incompatibility of the polymer thin films with DMSOperovskite deposition. Indeed, employing a protective later for the DBR during the perovskite deposition allows more homogeneous sample and larger finesse (See schematic in Figure 1c). In this case high surface tension perfluorinated polymer with low wettability protects the commercial polymers and avoid their dissolution.[27] On the other hand, such low wettability implies a surface activation process to deposit the perovskite thin film on the protective layer, complicating the fabrication process.[27] The spectra of Figure 1c' show the reflectance collected on different part of the sample surface for a microcavity made of CA and $\mathrm{PVK}$ where the cavity layer is made of $\mathrm{MAPbI}_{3}$ casted from DMSO solution like in the previous case. The sample is again very homogenous and present better optical quality with respect to the microcavity fabricated without protective layers, as testified by the superimposition of the data and by the interference patter. All the spectra show a relatively broad PBG peaked at about $810 \mathrm{~nm}$. The intensity of the reflected light in this case is larger than $100 \%$ because the sample reflects more light than the aluminium mirror employed as reference for the measurements. The peak shows a cavity mode positioned on the short wavelength side, that indices major variations in the perovskite emission. The $\mathrm{MAPbI}_{3}$ luminescence ranges from $710 \mathrm{~nm}$ and 820 $\mathrm{nm}$ and is peaked at $760 \mathrm{~nm}$. When coupled with the microcavity it results strongly modulated and a cavity mode is detected at $780 \mathrm{~nm}$ (Figure 1c"'). Such feature is sharp and corresponds to a finesse of 105 , which is the best reported for polymer microcavities containing perovskites thin films.[27]

These data clearly show that the DMSO processing is an issue for the implementation of perovskite film within polymer structure, this solvent can indeed dissolve polymers with both polar and non-polar characters, affecting the homogeneity and in turn the performances of the photonic structure. On the other hand, the possibility to fabricate device for single color enhancement or suppression and for lasing by a start-toend solution processing is highly interesting for the simplification of fabrication processes and for their cost reduction. The data reported in this work, demonstrates that employing specialty polymers as protective coating 
it is possible to obtain photonic structure able $t$ modify the emission successfully. On the other hand, these materials require processes of surface activation that make the growth of the structure more complex.

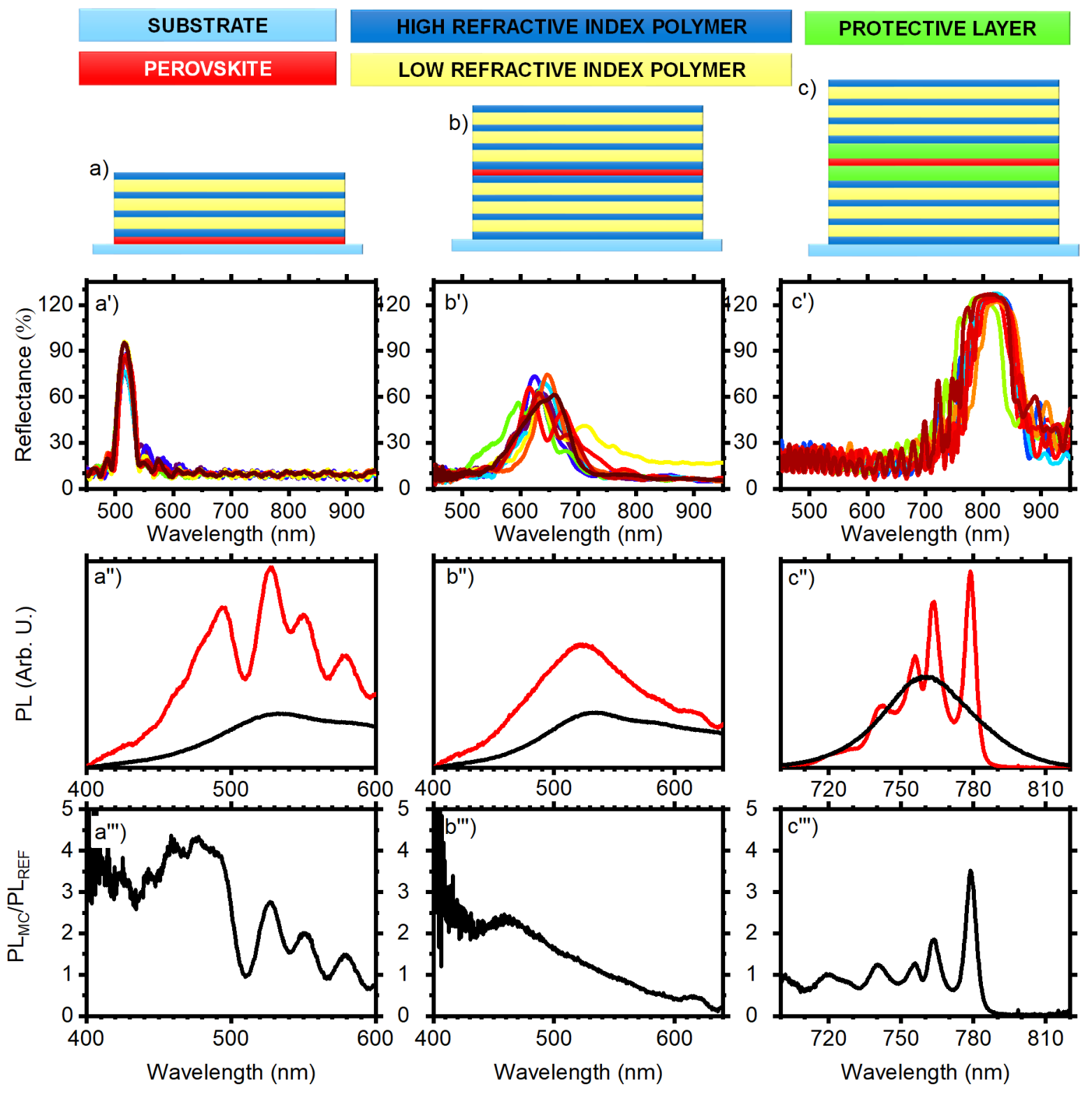

Fig. 1. Schematic (a, b, c) reflectance spectra (a', b', c') photoluminescence spectra of the photonic structure (red line) and of the reference film (black line) (a", b", c"), and ratio spectra calculated normalizing the photonic structure emission by the reference emission (a", , b"' c"') for the three architectures: (EDBE) $\mathrm{PbCl}_{4}$ coupled with a DBR[8] (panels a) and with a full microcavity (panels b) and $\mathrm{MA} \mathrm{PbCl}_{4}$ embedded in a microcavity employing a protective layer.[27]

\section{Conclusions}

In Summary, we reported on all polymer planar photonic structure embedding hybrid perovskite thin films with different architectures, including a DBR and two microcavities made with and without the use of a protective layers. In general, when the perovskite-DMSO solution is cast on the substrate or on a protective layer, it is possible to obtain structures with good optical quality and homogeneity, on the other hand, the direct deposition of the perovskite solution on the DBR polymers induces their dissolution and then the formation of inhomogeneous structures affected by many defects.

\section{References}

1. Deschler, F.; Price, M.; Pathak, S.; Klintberg, L.E.; Jarausch, D.-D.; Higler, R.; Hüttner, S.; Leijtens, T.; Stranks, S.D.; Snaith, H.J., et al., J. Phys. Chem. Lett., 5, 1421-1426, (2014).

2. Cho, H.; Jeong, S.-H.; Park, M.-H.; Kim, Y.-H.; Wolf, C.; Lee, C.-L.; Heo, J.H.; Sadhanala, A.; 
Myoung, N.; Yoo, S., et al., Science, 350, 12221225, (2015).

3. Cortecchia, D.; Mróz, W.; Neutzner, S.; Borzda, T.; Folpini, G.; Brescia, R.; Petrozza, A., Chem., 5, 2146-2158, (2019).

4. Saliba, M.; Correa-Baena, J.-P.; Grätzel, M.; Hagfeldt, A.; Abate, A., Angew. Chem. Int. Ed., 57, 2554-2569, (2018).

5. Cortecchia, D.; Neutzner, S.; Yin, J.; Salim, T.; Srimath Kandada, A.R.; Bruno, A.; Lam, Y.M.; Martí-Rujas, J.; Petrozza, A.; Soci, C., APL Materials, 6, 114207, (2018).

6. Park, H.J.; Xu, T.; Lee, J.Y.; Ledbetter, A.; Guo, L.J., ACS Nano, 5, 7055-7060, (2011).

7. Cortecchia, D.; Yin, J.; Bruno, A.; Lo, S.-Z.A.; Gurzadyan, G.G.; Mhaisalkar, S.; Bredas, J.-L.; Soci, C., J. Mater. Chem. C, 5, 2771-2780, (2017).

8. Lova, P.; Cortecchia, D.; S. Krishnamoorthy, H.N.; Giusto, P.; Bastianini, C.; Bruno, A.; Comoretto, D.; Soci, C., ACS Photonics, 5, 867-874, (2018).

9. Dohner, E.R.; Jaffe, A.; Bradshaw, L.R.; Karunadasa, H.I., J. Am. Chem. Soc., 136, 1315413157, (2014).

10. Dou, L.; Wong, A.B.; Yu, Y.; Lai, M.; Kornienko, N.; Eaton, S.W.; Fu, A.; Bischak, C.G.; Ma, J.; Ding, T., et al., Science, 349, 1518-1521, (2015).

11. Yin, J.; Li, H.; Cortecchia, D.; Soci, C.; Brédas, J.L., ACS Energy Lett., 2, 417-423, (2017).

12. Cortecchia, D.; Neutzner, S.; Srimath Kandada, A.R.; Mosconi, E.; Meggiolaro, D.; De Angelis, F.; Soci, C.; Petrozza, A., J. Am. Chem. Soc., 139, 3942, (2017).

13. Cortecchia, D.; Yin, J.; Petrozza, A.; Soci, C., J. Mater. Chem. C, 7, 4956-4969, (2019).

14. Cortecchia, D.; Lew, K.C.; So, J.-K.; Bruno, A.; Soci, C., Chem. Mater., 29, 10088-10094, (2017).

15. Paternò, G.M.; Moscardi, L.; Donini, S.; Ariodanti, D.; Kriegel, I.; Zani, M.; Parisini, E.; Scotognella, F.; Lanzani, G., J. Phys. Chem. Lett., 10, 49804986, (2019).

16. Paternò, G.M.; Iseppon, C.; D’Altri, A.; Fasanotti, C.; Merati, G.; Randi, M.; Desii, A.; Pogna, E.A.A.; Viola, D.; Cerullo, G., et al., Sci. Rep., 8, 3517, (2018).

17. Paternò, G.M.; Moscardi, L.; Kriegel, I.; Scotognella, F.; Lanzani, G., SPIE Proc., 8, 1-8, (2018).

18. Lova, P.; Manfredi, G.; Bastianini, C.; Mennucci, C.; Buatier de Mongeot, F.; Servida, A.; Comoretto, D., ACS Appl. Mater. Interfaces, 11, 16872-16880, (2019).

19. Giusto, P.; Lova, P.; Manfredi, G.; Gazzo, S.; Srinivasan, B.; Radice, S.V.; Comoretto, D., ACS Omega, 3, 7517-7522, (2018).

20. Lova, P., Polymers, 10, 1161, (2018).

21. Lova, P.; Bastianini, C.; Giusto, P.; Patrini, M.; Rizzo, P.; Guerra, G.; Iodice, M.; Soci, C.; Comoretto, D., ACS Appl. Mater. Interfaces, 8, 31941-31950, (2016).

22. Iasilli, G.; Francischello, R.; Lova, P.; Silvano, S.; Surace, A.; Pesce, G.; Alloisio, M.; Patrini, M.;
Shimizu, M.; Comoretto, D., et al., Mater. Chem. Front., 3, 429-436, (2019).

23. Manfredi, G.; Lova, P.; Di Stasio, F.; Rastogi, P.; Krahne, R.; Comoretto, D., RSC Advances, 8, 13026-13033, (2018).

24. Fornasari, L.; Floris, F.; Patrini, M.; Comoretto, D.; Marabelli, F., Phys. Chem. Chem. Phys., 18, 1408614093, (2016).

25. Lova, P.; Grande, V.; Manfredi, G.; Patrin, M.; Herbst, S.; Würthner, F.; Comoretto, D., Adv. Opt. Mater., 5, 1700523, (2017).

26. Manfredi, G.; Lova, P.; Di Stasio, F.; Krahne, R.; Comoretto, D., ACS Photonics, 4, 1761-1769, (2017).

27. Lova, P.; Giusto, P.; Di Stasio, F.; Manfredi, G.; Paternò, G.M.; Cortecchia, D.; Soci, C.; Comoretto, D., Nanoscale, 11, 8978-8983, (2019).

28. Lova, P.; Manfredi, G.; Comoretto, D., Adv. Opt. Mater., 6, 1800730-1800726, (2018). 